\title{
Monitoring brain activity of human subjects during delayed matching to sample tasks comparing verbal and pictorial stimuli with modal and cross-modal presentation: an event related potential study employing a source reconstruction method
}

\author{
Christian Dobel ${ }^{a, *}$, Olaf Hauk ${ }^{a}$, Elvira Zobela ${ }^{a}$ Carsten Eulitz ${ }^{a}$, Friedemann Pulvermüller ${ }^{a}$, \\ Rudolf Cohen ${ }^{a}$, Paul Walter Schönle ${ }^{a, b}$, Thomas Elbert ${ }^{a}$, Brigitte Rockstroh ${ }^{\mathrm{a}, \mathrm{b}}$ \\ ${ }^{a}$ Department of Psychology, PF 5560-D23, University of Konstanz, Universitätsstrasse 10, D-78434 Konstanz, Germany \\ ${ }^{b}$ Lurija-Institute for Rehabilitation and Health Research, Schmieder Rehabilitation Hospital, Allensbach, Germany
}

Received 5 June 1998; received in revised form 24 July 1998; accepted 3 August 1998

\begin{abstract}
The time course of the event related potentials evoked within a delayed matching to sample task employing verbal and pictorial stimuli was analyzed with a source reconstruction method (miminum norm method). During signal stimulus presentation pictorial stimuli evoked more activity than verbal stimuli. Activity was particularly prominent in left frontal areas for the match of verbalverbal stimulus pairs and over right posterior regions for the match of verbal-pictorial stimuli. Anticipation of the to-be-matched stimulus produced more pronounced activity for pictorial stimuli and generally stronger left and frontal activity. Results are discussed referring to a biological model of language processing. () 1998 Elsevier Science Ireland Ltd. All rights reserved
\end{abstract}

Keywords: Event related potentials; Minimum norm; Source reconstruction; Language; Delayed-matching-to-sample; Token test

Two-stimulus-reaction time and delayed-matching-tosample tasks have often been used to examine frontal lobe functions in healthy subjects and patients with psychiatric or neurological disorders [11]. The basic design usually comprises a first stimulus (S1) that signals the presentation of a second 'imperative', i.e. response-relevant stimulus (S2) to follow after a distinct time interval.

The same stimulus structure underlies a neuropsychological test, the so called 'Token Test' [5], which has proven to differentiate reliably between aphasic patients and other neurological patients [7]. In one part of the test, plastic tokens varying in color, form and size are placed in front of the patient who is asked to point at a specific token according to the instruction (e.g. 'Point at the large red circle!'). Previous studies found that successful test performance, in addition to auditory comprehension, requires 'the

\footnotetext{
* Corresponding author. Tel.: +49 7531 884604; fax: +49 7531 884601; e-mail: christian.dobel@uni-konstanz.de
}

analytical isolation and the cognitive handling of individual features of concepts' $[2,3]$. In the present study, the Token Test was realized within the two-stimulus reaction time paradigm in order to examine event-related brain potentials related to these cognitive and language-related processes in healthy subjects as well as in patients recovering from aphasia. The results of a group of healthy subjects are reported in the present paper.

In close resemblance to the Token Test a noun phrase describing an object was presented for $1.5 \mathrm{~s}$ as $\mathrm{S} 1$, which was followed after an interstimulus interval of $2 \mathrm{~s}$ by the presentation of the pictures of two objects as S2. Objects were defined on three dimensions: color, form and size. Only one of the two objects presented as S2 matched the verbal description provided in the $\mathrm{S} 1$ on all three dimensions. Subjects were asked to indicate, by pressing a button with the index or middle finger of the left hand, whether the left or the right picture in S2 matched the description provided in the $\mathrm{S} 1$. The button press terminated the stimulus presentation; the interval until the next $\mathrm{S} 1$ was $3 \mathrm{~s}$. 
Within four series of 48 trials each, the effects of type of stimulus and type of task on the scalp distribution of the event-related potentials (ERPs) were examined, in that the $\mathrm{S} 1$ comprised either the verbal description or the pictorial display of objects, and in that either matching of pictorial S2 to verbal S1 and vice versa (cross modal match) or matching the same stimulus types (intra modal match) were required. The sequence of trial blocks/conditions was counterbalanced across subjects. Subjects were verbally instructed about each task. Practice trials assured that instructions were adequately understood.

Fourteen healthy subjects (four women, mean $40 \pm 8.8$ years) with high school graduation were paid for participation in the study. All subjects were right handers according to the Edinburgh Inventory [8].) The verbal S1 comprised vertically arranged four-word-descriptions of objects that varied on three dimensions, form (star or circle), size (large, small) and color (blue, yellow, e.g. 'The small blue star'). Two-step variation of all three dimensions resulted in 24 combinations altogether. Each combination was presented twice within the total 48 trials of each condition. The pictorial S1 comprised the respective object drawing (e.g. a small blue star). The verbal S2 consisted of two horizontally aligned words expressing one of the three dimensions (e.g. star-circle), while two object drawings aligned horizontally next to each other comprised the pictorial S2. Stimuli were generated by the STIM system (NEUROSCAN) and presented on a 14-inch monitor (visual angle: 8.6). Response buttons were easily manageable microswitches integrated into a key pad mounted on the left arm-rest of the subject's chair.

The EEG was recorded from $26 \mathrm{Sn}$ electrodes (including Fz, Cz, Pz, Fp1, C3, F3, F7, T3, T5, P3, O1, Fp2, C4, F4, F8, T4, T6, P4, O2, and both mastoids, M1, M2) mounted on an electrode cap (Electrocap). The vertical and horizontal EOG were recorded for the correction of eye movement artifacts on ERPs with two electrodes placed about $1 \mathrm{~cm}$ below the eyes, one on the forehead between the eyes, and two electrodes placed on the outer canthi horizontally to the eyes. Electrode impedances were kept below $5 \mathrm{k} \Omega$. A DC-amplifier (MES, Munich) served for data acquisition. Bandwidth ranged from $\mathrm{DC}$ to $30 \mathrm{~Hz}$ (6 dB/octave). Data were digitized at $1 \mathrm{bin} / \mu \mathrm{V}, 16$-bit A/D, and sampled at $100 \mathrm{~Hz}$. Data were recorded continuously and stored for off-line analysis. A calibration run for eye movement correction preceded the experiment. Following the recording, electrode positions were digitized in three dimensions using a 3D digitizer (Polhemus).

The continuously recorded data were first corrected for slow DC shifts by polynomial correction using means in relation to markers over the whole recording. The continuous data were then transformed into epochs $1000 \mathrm{~ms}$ before and $14000 \mathrm{~ms}$ after S1. Slow shifts were corrected by subtracting a linear trend from each channel on each trial. Data were then baseline corrected with $500 \mathrm{~ms}$ before S1 and transformed to average reference. Epochs were corrected for eye movement and blink artifacts following the method of Berg and Scherg [1] that allows for the use of the electrodes around the eyes as EEG electrodes after correction. This method distinguishes between ocular and brain activity and corrects for ocular artifacts only. After correction, each trial was visually inspected and excluded if there were remaining artifacts of any kind (muscle potentials, large drifts, etc.). The remaining artifact-free trials were averaged across conditions and filtered with a $5 \mathrm{~Hz}$ low pass filter.

The ERP time course between S1- and S2-onset was analyzed for successive $500 \mathrm{~ms}$ intervals. The typical VEP response to the onset of the visual $\mathrm{S} 1(\mathrm{P} 1, \mathrm{P} 3)$ was examined by the additional analysis of the interval $200-400 \mathrm{~ms}$ following S1-onset.

Fig. 1 illustrates the time course of the ERP (grand mean) from S1 onset to S2 onset for the different conditions; the VEP as well as the development of a slow negative shift preceding the imperative S2 (a contingent negative variation, CNV) become obvious.

In complex cognitive tasks such as the present one, we have no a priori knowledge about the number, extent or approximate locations of the underlying brain sources. Therefore, a source reconstruction method was considered in order to account for the fact that the signal measured at an electrode is the summation of the signals arising from sources distributed over a large area of the brain, and that a source does not necessarily produce maximal signal intensity on the scalp directly above its true location in the brain. For that reason a method was chosen that only requires the information contained in the measured data to make inferences about the current distribution giving rise to the measured potential. Such a solution is obtained with the 'pseudoinverse' or 'minimum norm method'; given a large amount of nearly spaced dipoles with fixed locations and orientations, which shall approximate the whole brain volume, and the physical relationship between each of these sources and the potential produced by them, this method yields the closest mapping of the measured potential towards the underlying brain sources in the absence of any a priori information. This results in a linear transformation of the measured potential, i.e. the source strength of each of the fixed dipoles is estimated by multiplying the data with a spatial filter vector that has optimal resolution properties [6]. As this method is not based on isolated dipoles, the requirements for its application are met also in the presence of large brain noise. However, since it is hard to statistically handle this amount of data for all dipoles, and since the resolving power of the present data set does not allow a precise and independent estimate for every source strength, we focused only on the dipole strengths for locations under the electrodes. By this, we got a rough twodimensional projection of the real current distribution. The pitfalls of this method are that amplitudes of deeper sources are underestimated compared with more superficial ones, and the amplitudes of tangential sources are underestimated compared with radial ones. In addition, the solu- 


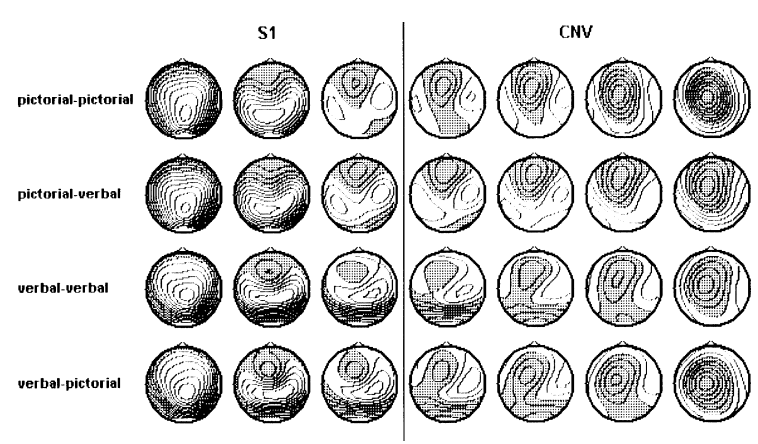

Fig. 1. ERP spline maps (grand mean, average reference, grey denoting negativity, $0.5 \mu \mathrm{V}$ per contour line) of the experimental conditions from onset of S1 to onset of S2 averaged across steps of $500 \mathrm{~ms}$ (first column: $200-400 \mathrm{~ms}$ ).

tion for a single point source is widely blurred. However, these problems are inherent in the measurement of potentials at the surface of the head, and in effect, the resolution properties of our method can be considered to be significantly better than those of raw potentials.

The minimum norm $(\mathrm{MN})$ values were taken as indicators of the area-specific amount of activation. Effects of stimulus and task conditions on the distribution of these activity indices over the two hemispheres and anterior and posterior areas were examined by comparing four areas (left anterior: F9, Fp1, F7, F3, C3; right anterior: F10, Fp2, F8, F4, C4; left posterior: $\mathrm{P} 3, \mathrm{~T} 7, \mathrm{P} 7, \mathrm{M} 1, \mathrm{O} 1$; right posterior: $\mathrm{P} 4, \mathrm{~T} 8, \mathrm{P} 8$,
M2, O2) with an analysis of variance (ANOVA) with the within-subjects factors MODALITY (verbal vs. pictorial), TASK (intra modal vs. cross modal), GRADIENT (anterior vs. posterior areas), HEMISPHERE (left vs. right areas) and SEGMENT (successive 500-ms intervals between S1-onset and S2-onset). A combination of electrodes was chosen to im-prove the signal-to-noise ratio. $P$-Values were determined after adjusting the degrees of freedom with the Greenhouse-Geisser-Epsilon.

Fig. 2 displays the MN values at the four selected areas and across the S1-S2 interval for the four experimental conditions. A pronounced posterior activity is exhibited during the interval in which the VEP is to be expected (200-400 $\mathrm{ms}$ ); this contribution of the VEP (comprising P1 and P3) is documented by the main effect GRADIENT $(F(1,13)=$ $21.2, P<0.0005)$. Interestingly, the type of stimulus also affected the activity in this early time segment in that pictorial stimuli evoked more pronounced activity than verbal stimuli (MODALITY $(F(1,13)=5.8, P<0.03)$. We may speculate that the pictorial stimuli were more complex and therefore provoked more activity than the verbal stimuli.

Given that one syllable can be processed every $200 \mathrm{~ms}$ [9], we suppose that in the first time interval only the denominator of the noun phrase, i.e. a high frequent and constant and therefore less complex item, was processed.

The posterior predominance of activity prevailed throughout the subsequent $500-\mathrm{ms}$ interval of the S1 pre-

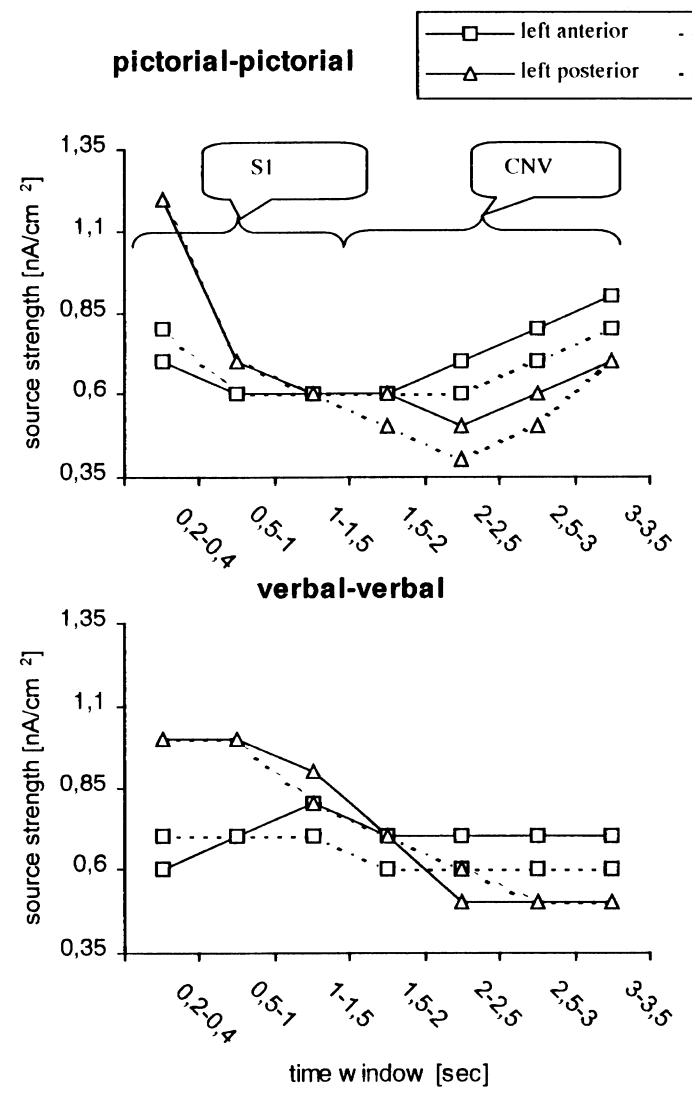

$\ldots \square-\cdots$ right anterior
$\cdots \Delta \cdots$ right posterior

pictorial-verbal
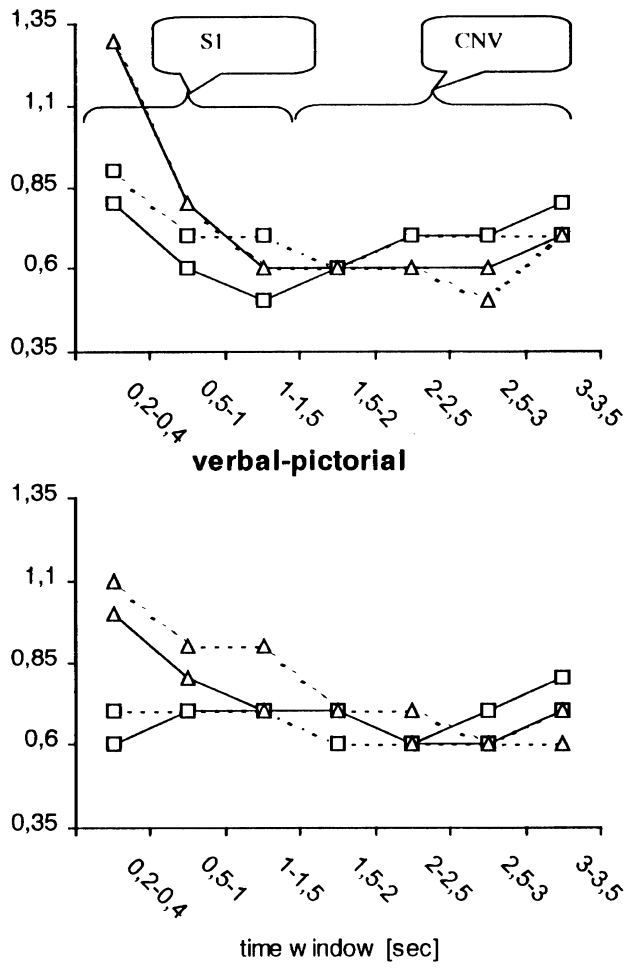

Fig. 2. Minimum norm values (source strength) of the experimental conditions from onset of S1 to onset of S2 averaged across steps of $500 \mathrm{~ms}$ (first step: 200-400 ms). 
sentation, as indicated by the main effect GRADIENT ( $F$ $(1,13)=5.9, P<0.03)$, while by the end of $\mathrm{S} 1$-interval $(1.0-1.5 \mathrm{~s})$, stimulus-modality as well as the signaled task affected the scalp distribution of activity. An interaction TASK $\times$ HEMISPHERE $(F(1,13)=8.6, P<0.01)$ confirmed more pronounced left-hemispheric activity whenever the signal and imperative stimuli were of the same modality (verbal-verbal or pictorial-pictorial) compared to crossmodal conditions, while the opposite pattern of activity emerged in the right hemisphere. Furthermore, verbal stimuli produced more left-anterior activity $(F(1,13)=15.2$, $P<0.002)$, while verbal stimuli signaling a match/mismatch decision with pictorial stimuli generated activity in the right-posterior area (MODALITY $\times$ GRADIENT $\times$ HEMISPHERE, $F(1,13)=7.9, P<0.01)$.

This pattern suggests that the right hemisphere is especially called upon when a transformation of one mode of representation into another one takes place. If, on the other hand, the S2 is of verbal nature, the information about the correct word form in the S1 may be needed and perisylvian word form assemblies remain active. This hypothesis refers to a biological conceptualization of language processing [9] with sequences of cell assemblies, each taking approximately $200 \mathrm{~ms}$ for its 'ignition'. If the number of assemblies corresponds to the number of syllables of the input string, an activation time of roughly $1200 \mathrm{~ms}$ would result. During this interval, the amount of neuronal activity would increase because ignited assemblies may 'retain' their activity for some time before they are deactivated again. The increasing number of simultaneously active assemblies would explain the more pronounced activity in the verbal-verbal condition.

In summary, stimulus-evoked cortical activity is not only determined by the stimulus modality (visual) but already affected by the stimulus meaning (verbal, pictorial) and the anticipated task [10]. The change in the activity pattern across the S1-interval (SEGMENT $\times$ MODALITY $(F(2,26)=12.4, \epsilon=0.66, P<0.001$; SEGMENT $\times$ MODALITY $\times$ GRADIENT $\times \operatorname{HEMISPHERE~}(F(2,26)=6.7, \epsilon=$ $0.77, P<0.01)$ suggests task-oriented stimulus processing taking place during S1-presentation.

Task-related activation, as indicated by the $\mathrm{MN}$ values during the last $500 \mathrm{~ms}$ preceding the S2, corresponding to the $\mathrm{CNV}$, turned out to be more pronounced for objects than for words (MODALITY $F(1,13)=4.4 ; P<0.05)$. This effect emphasizes the demands of pictorial matching upon verbal instructions, which may explain the particular deficits of aphasics in an entirely non-verbal version of the Token Test [4]. In contrast to the activity pattern during $\mathrm{S} 1$, activity preceding the $\mathrm{S} 2$ predominated over anterior areas (GRADIENT $F(1,13)=12,0 ; P<0.01)$ and the left hemisphere (HEMISPHERE $F(1,13)=8,2 ; P<0.01$ ). S2 complexity (three dimensions are displayed in the pictorial stimuli, while the verbal stimuli refer only to one dimension) did not affect the activity pattern during this time interval. The left-hemispheric predominance of activity cannot be attributed to motor response preparation, as subjects responded with their left hand. Two processes may be assumed during the anticipatory (CNV-) interval [11]: one unspecific process that is marked by frontal activation and one task specific process that is related to the demands of the task at hand. The left- and anterior predominance of the $\mathrm{CNV}$-corresponding MN pattern suggests that the "the analytical isolation and the cognitive handling of individual features of concepts' and the preparation for matching stimuli according to the instruction constitutes a lateralized process, and that the stronger left hemispheric activity reflects the activity of components that are particularly involved in operations necessary to solve the Token Test and similar tasks.

Research was supported by the Deutsche Forschungsgemeinschaft.

[1] Berg, P. and Scherg, M., A multiple source approach to the correction of eye artifacts, Electroenceph. clin. Neurophysiol., 90 (1994) 229-241.

[2] Cohen, R., Glöckner, A., Lutz, M., Maier, T. and Meier, E., Cognitive impairments in aphasia: new results and new problems. In R. Bäuerle, C. Schwarze and A. von Stechow (Eds.), Meaning, Use, and Interpretation of Language, De Gruyter, Berlin, 1983, pp. 30-45.

[3] Cohen, R., Kelter, S. and Woll, G., Analytical competence and language impairment in aphasia, Brain Lang., 10 (1980) 331347.

[4] Cohen, R., Lutzweiler, W. and Woll, G., Zur Konstruktvalidität des Token Test, Nervenarzt, 51 (1980) 30-35.

[5] De Renzi, E. and Vignolo, L.A., The Token Test: a sensitive test to detect receptive disturbances in aphasics, Brain, 85 (1962) 665-678.

[6] Grave de Peralta Menendez, R., Hauk, O., Gonzalez Andino, S.L., Vogt, H. and Michel, C., Linear inverse solutions with optimal resolution kernels applied to the electromagnetic tomography, Human Brain Mapping, 5 (6) (1997) 454-467.

[7] Huber, W., Poeck, K., Weniger, D. and Willmes, K., Aachener Aphasie Test, Verlag für Psychologie, Hogrefe, Göttingen, 1983.

[8] Oldfield, R.C., The assessment and analysis of handedness: the Edinburgh Inventory, Neuropsychologia, 9 (1971) 97-113.

[9] Pulvermüller, F., Constituents of a neurological theory of language, Concepts in Neuroscience, 3 (2) (1992) 157-200.

[10] Rockstroh, B., Elbert, T., Canavan, A., Lutzenberger, W. and Birbaumer, N., Slow Cortical Potentials and Behaviour, Urban und Schwarzenberg, Baltimore, MD, 1989, 99 pp.

[11] Walter, W.G., Electric signs of expectancy and decision in the human brain. In H.L. Oestreicher and D.R. Moore (Eds.), Cybernetic Problems in Bionics, Gordon and Breach, New York, 1968, pp. 361-396. 